\title{
Relación entre el índice lipoproteínas de baja densidad (LDL)/lipoproteínas de alta densidad (HDL) con enzimas antioxidantes y el índice oxLDL/HDL
}

\author{
Irma Zamora-Ginez, ${ }^{*}$ Blanca Guadalupe Baez-Duarte, ${ }^{1}$ Adriana Nieva-Vazquez, ${ }^{2}$ Karla Hilsen García-Aragón, ${ }^{3}$ \\ Eduardo Monjaraz-Guzmán, ${ }_{4}^{4}$ Francisco Mendoza-Carrera, ${ }^{5}$ Paola Meneses-Zamora, ${ }_{1}$ \\ Claudia Valeria Flores-Blanco ${ }^{1}$ y Karina Luna-De Jesús ${ }^{6}$ \\ ${ }^{1}$ Benemérita Universidad Autónoma de Puebla, Facultad de Medicina, Puebla; ${ }^{2 B}$ Benemérita Universidad Autónoma de Puebla, Complejo Regional \\ Sur, Puebla; ${ }^{3}$ Universidad Autónoma del Estado de Morelos, Morelos; ${ }^{4}$ Benemérita Universidad Autónoma de Puebla, Unidad de Fisiología, Puebla; \\ ${ }^{5}$ Instituto Mexicano del Seguro Social, Centro de Investigación Biomédica de Occidente, División de Medicina Molecular, Jalisco; ${ }^{6}$ Instituto Politécnico \\ Nacional, Centro de Investigación en Biotecnología Aplicada, Tlaxcala. México
}

\section{Resumen}

Introducción: El índice de lipoproteínas de baja densidad (LDL)/lipoproteínas de alta densidad (HDL) es un factor predictivo de aterosclerosis, la cual está asociada con modificaciones oxidativas. Objetivo: Evaluar la asociación del índice con marcadores de estrés oxidativo. Método: Se incluyeron 444 sujetos, caracterizados clínica, antropométrica y bioquímicamente; se cuantificó superóxido dismutasa, glutation peroxidasa 3 (GPX3), magnesio e índice LDL oxidadas (oxLDL/HDL). Resultados: La disminución en 1.014 unidades del índice $L D L / H D L$ se asoció con aumento de 1 unidad/mL de superóxido dismutasa $(p=0.030)$ y la de 0.023 unidades con aumento de $1 \mathrm{nmol} / \mathrm{minuto} / \mathrm{mL}$ de GPx3 $(p<0.0005)$. El aumento en 1 unidad del índice se asoció con aumento de 0.831 unidades en el índice oxLDL/HDL ( $p<0.05)$. Después de controlar el efecto del sexo, edad, fumar, obesidad y resistencia a la insulina, la reducción de 0.001 por unidad del índice se asoció con aumento de $1 \mu \mathrm{g} / \mathrm{g}$ de magnesio en uñas $(p=0.020)$. Conclusiones: El índice $L D L / H D L$ presenta relación inversa con el estado antioxidante y relación directa con el estado de oxidación, independientemente de otros factores de riesgo cardiovascular y de estrés oxidativo.

PALABRAS CLAVE: Índice LDL/HDL. Antioxidantes. Índice oxLDL/HDL.

\begin{abstract}
Introduction: The low-density lipoprotein (LDL)/high-density lipoprotein (HDL) index is a predictive factor for atherosclerosis, which is associated with oxidative modifications. Objective: To assess the association of the index with oxidative stress markers. Methods: 444 subjects were included and were clinically, anthropometrically and biochemically characterized; superoxide dismutase, glutathione peroxidase 3(GPX3), magnesium and oxidized LDL (oxLDL) index (oxLDL/HDL) were quantified. Results: $A$ decrease of 1.014 units in the LDL/HDL index was associated with a superoxide dismutase increase of 1 unit $/ \mathrm{mL}(p=0.030)$, while a decrease of 0.023 units was associated with a GPx3 increase of $1 \mathrm{nmol} / \mathrm{min} / \mathrm{mL}(p<0.0005)$. An increase of one unit in the index was associated with an increase of 0.831 in the oxLDL/HDL index $(p<0.05)$. After controlling for the effect of gender, age, smoking, obesity and insulin resistance, a reduction of 0.001 per index unit was associated with an increase of $1 \mu \mathrm{g} / \mathrm{g}$ of magnesium in the nails $(p=0.020)$. Conclusions: The $L D L / H D L$ index shows an inverse relationship with the antioxidant status and a direct relationship with oxidation status, regardless of other cardiovascular and oxidative stress risk factors.
\end{abstract}

KEY WORDS: Low-density lipoprotein/high-density lipoprotein index. Antioxidants. Oxidized low-density lipoprotein/high-density lipoprotein index.

Correspondencia:

*Irma Zamora-Gínez

E-mail: irma.zamora@correo.buap.mx
Fecha de recepción: 12-12-2018

Fecha de aceptación: 21-02-2019

DOI: 10.24875/GMM.19004921
Gac Med Mex. 2019;155:487-492

Disponible en PubMed

www.gacetamedicademexico.com 


\section{Introducción}

La oxidación lipídica es una de las principales causas de aterosclerosis: la lipoproteína de baja densidad (LDL, low-density lipoproteins), principal lipoproteína oxidativa, la lipoproteína de alta densidad (HDL, high-density lipoproteins) y la lipoproteína con actividad antioxidante. ${ }^{1-3}$ Recientemente, el índice LDL oxidada (oxLDL)/HDL se ha propuesto como predictor de estrés oxidativo en pacientes dializados y como biomarcador de enfermedad cardiovascular (ECV). ${ }^{4,5}$

El aumento de la oxidación lipídica se ha relacionado con mieloperoxidasa $(\mathrm{MPO})^{6}$ y deficiencia de magnesio. ${ }^{7}$ Por otra parte, la reducción del estrés oxidativo mediante las enzimas antioxidantes superóxido dismutasas (SOD) y glutatión peroxidasa 3 (GPx3) se asocia inversamente con ECV.,9

Actualmente, el índice LDL/HDL se ha propuesto como un indicador de riesgo de cardiovascular (RCV) con mayor valor predictivo que las lipoproteínas que lo forman, ${ }^{10-12}$ sin embargo, hasta nuestro conocimiento, no se ha explorado su relación con biomarcadores de estrés oxidativo, con la finalidad de establecer si pudiera considerarse referencia temprana para el estado oxidativo.

En general, este estudio tuvo como objetivo evaluar la asociación del índice LDL/HDL con SOD, GPx3, MOP, magnesio y oxLDL/HDL, después de controlar el efecto del sexo, edad, consumo de tabaco, obesidad y resistencia a la insulina, parámetros que se han asociado con ECV o estrés oxidativo. ${ }^{13}$

\section{Método}

El estudio se realizó de acuerdo con la Declaración de Helsinki. Los participantes dieron su consentimiento informado por escrito. Los resultados son parte de protocolo 342, aprobado por el Comité de Investigación de la Facultad de Medicina, Benemérita Universidad Autónoma de Puebla. Diseñamos un estudio transversal en voluntarios hombres y mujeres no embarazadas, de 18 a 65 años, de enero a diciembre de 2017. Se excluyeron sujetos con dependencia al cigarrillos $^{14} \mathrm{y} / \mathrm{o}$ alcohol ${ }^{15} \mathrm{y} / \mathrm{o}$ con consumo de suplementos vitamínicos en el último año, y/o con ingesta de esteroides o medicamentos que interfieren con el metabolismo de los lípidos, así como los sujetos con ECV, enfermedad renal, enfermedad hepática o enfermedad inflamatoria.

\section{Caracterización clínica y antropométrica}

Se realizó historia clínica estandarizada. Con el sujeto en condiciones de ayuno, con ropa liviana y sin zapatos, se midió talla y peso, con los cuales se calculó el índice de masa corporal (IMC) como parámetro de obesidad (peso en kilogramos/altura en $\mathrm{m}^{2}$ ). Se consideró consumo de cigarrillos bajo, moderado y alto. ${ }^{14}$

\section{Ensayos}

Las muestras de sangre se extrajeron en el laboratorio del Hospital Universitario de Puebla, mediante punción venosa después de 12 horas de ayuno nocturno. Glucosa, insulina, triglicéridos, colesterol total, LDL, HDL y magnesio sérico se determinaron mediante métodos estandarizados. La lipoproteína de muy baja densidad (VLDL) se estimó dividiendo triglicéridos entre 5. La resistencia a la insulina se cuantificó mediante HOMA-IR (homeostatic model assessment insulin resistance) con base en glucosa en ayuno $(\mathrm{mmol} / \mathrm{L}) \times$ insulina en ayunas $(\mu \mathrm{U} / \mathrm{mL}) / 22.517$, mediante la calculadora HOMAC. ${ }^{16}$ Siguiendo las instrucciones de kits comerciales se determinaron SOD, GPx3, MPO y oxLDL. La concentración de magnesio de muestras de uñas de pies y manos se midió por espectrometría de absorción atómica.

La normalidad se probó mediante la prueba de Kolmogorov-Smirnov. Las diferencias entre grupos (RCV: LDL/HDL > 3.0 $)^{17}$ se estimaron utilizando $U$ de Mann-Whitney para variables numéricas y la prueba exacta de Fisher para variables cualitativas. Se utilizó regresión lineal multivariable y coeficiente de correlación parcial para determinar las asociaciones ajustadas para edad (años), sexo (femenino/masculino), fumador (no/sí), IMC $\left(\mathrm{kg} / \mathrm{m}^{2}\right)$ y HOMA-IR. Se utilizó el programa estadístico SPSS, versión 23.0; $p<0.05$ fue considerado estadísticamente significativo. Dado que MPO, magnesio en uñas y oxLDL/HDL se midieron en un número menor de participantes (74, 74 y 214 , respectivamente), se probó a posteriori la potencia estadística (1- $\beta$ ), utilizando el tamaño del efecto f2 (basado en valores $\mathrm{r}^{2}$ ajustados), mediante $G$ * Power, versión 3.0.10.

\section{Resultados}

En este estudio participaron 444 sujetos, con mayor participación de mujeres que de hombres 
(62.8 versus $37.2 \%, p=0.005)$. La caracterización de la población en estudio conforme LDL/HDL $(>3.0$ $\mathrm{RCV}$ ) se muestra en la Tabla 1. El análisis de regresión lineal y el coeficiente de correlación parcial mostraron que la actividad de SOD y GPx3 fueron predictores negativos de LDL/HDL, así como la concentración de magnesio en uñas después de ajustar para edad, sexo, fumar, obesidad y resistencia a la insulina (Tabla 2).

El índice LDL/HDL se ha definido en un intento por optimizar la capacidad predictiva del perfil lipídico, por lo que la tendencia de los componentes del índice fue probada para las variables con asociación significativa. SOD no se asoció con LDL (coeficiente $\beta$ [intervalo de confianza de $95 \%$, IC $95 \%]=5.662[-25.84$ $\left.37.17, r_{123456}=-0.015, p=0.724\right)$, pero sí positivamente con HDL (coeficiente $\beta$ [IC $95 \%$ ] $=21.550$ [12.237$\left.30.864], r_{123456}=-0.186, p<0.0005\right)$. GPx3 se asoció negativamente con LDL (coeficiente $\beta$ [IC 95 $\%]=-0.360$ [-0.545 a -0.174$], r_{123456}=-0.232$, $\mathrm{p}<0.0005$ ) y positivamente con HDL (coeficiente $\beta$ [IC $95 \%]=0.294$ [0.162-0.425], $r_{123456}=0.265$, $p<0.0005)$. El magnesio en uñas no se asoció con LDL (coeficiente $\beta$ [IC $95 \%]=-0.019$ [-0.079-0.41], $r_{123456}=-0.127, p=0.520$ ) ni con HDL (coeficiente $\beta$ [IC $95 \%]=0.012[-0.002-0.025], R_{123456}=0.333$, $p=0.084)$. Finalmente, el índice LDL/HDL se asoció directamente con el índice oxLDL/HDL (Tabla 3).

Dado que se midió MPO, magnesio de uña y oxLDL/ HDL en un número menor de participantes $(74,74$ y 240 , respectivamente) a posteriori probamos el poder estadístico de los análisis, el cual fue satisfactorio para todos los resultados (1- $\beta$ de $0.94,0.99$ y 0.80 , respectivamente).

\section{Discusión}

Mediante un estudio transversal analizamos la asociación del índice LDL/HDL con marcadores de estrés oxidativo, demostrando que se relaciona inversamente con la actividad de SOD y GPx3 y directamente con el índice oxLDL/HDL, relación que no es alterada por el efecto de la edad, el sexo, fumar, obesidad ni resistencia a la insulina.

Nuestro estudio demostró por primera vez que el aumento en 1 unidad $/ \mathrm{mL}$ en la actividad de SOD reduce el índice en 1.01 unidad, dependiendo aparentemente de un efecto adicional de modulación antioxidante entre SOD y HDL. Nuestros resultados coinciden con otros en los que se ha reportado correlación positiva entre SOD y éster hidrolasa
Tabla 1. Caracterización clínica, antropométrica y bioquímica de la población de estudio categorizada por el índice LDL/HDL $(<3.0$, riesgo cardiovascular)

\begin{tabular}{|c|c|c|c|}
\hline & $\begin{array}{c}\text { LDL/HDL } \leq 3.0 \\
(n=165)\end{array}$ & $\begin{array}{l}\text { LDL/HDL > } 3.0 \\
\quad(n=279)\end{array}$ & $p$ \\
\hline Sexo (F/M, \%) & $64.9 / 35.1$ & $48 / 52$ & $0.075^{\star}$ \\
\hline \multirow[t]{2}{*}{ Fumador (Sí, \%) } & 11.3 & 12.9 & $0.739^{*}$ \\
\hline & Media $\pm D E$ & Media $\pm D E$ & \\
\hline Edad (años) & $40.14 \pm 13.31$ & $42.68 \pm 11.75$ & $0.035^{\star *}$ \\
\hline Peso (kg) & $69.38 \pm 13.68$ & $75.86 \pm 14.88$ & $<0.0005^{\star \star}$ \\
\hline Talla (m) & $1.58 \pm 0.085$ & $1.59 \pm 0.09$ & $0.503^{* *}$ \\
\hline IMC (kg/m²) & $27.72 \pm 5.08$ & $29.89 \pm 5.08$ & $<0.0005^{\star *}$ \\
\hline $\begin{array}{l}\text { Glucosa de } \\
\text { ayudo (mg/dL) }\end{array}$ & $100.41 \pm 28.20$ & $107.76 \pm 34.37$ & $0.002^{* *}$ \\
\hline $\begin{array}{l}\text { Insulina de } \\
\text { ayuno }(\mu \mathrm{U} / \mathrm{mL})\end{array}$ & $10.41 \pm 8.44$ & $12.24 \pm 8.88$ & $0.005^{\star \star}$ \\
\hline HOMA-IR & $1.37 \pm 0.91$ & $1.52 \pm 0.96$ & $0.009^{\star \star}$ \\
\hline $\begin{array}{l}\text { Triglicéridos } \\
\text { (mg/dL) }\end{array}$ & $153.07 \pm 95.57$ & $192.34 \pm 83.32$ & $<0.0005^{\star \star}$ \\
\hline $\begin{array}{l}\text { Colesterol } \\
\text { total (mg/dL) }\end{array}$ & $185.91 \pm 37.44$ & $211.86 \pm 37.76$ & $<0.0005^{\star *}$ \\
\hline $\mathrm{HDL}$ (mg/dL) & $47.96 \pm 12.46$ & $36.81 \pm 8.56$ & $<0.0005^{\star *}$ \\
\hline LDL (mg/dL) & $88.04 \pm 41.66$ & $139.76 \pm 28.96$ & $<0.0005^{\star *}$ \\
\hline VLDL (mg/dL) & $30.61 \pm 19.11$ & $38.47 \pm 16.67$ & $<0.0005^{\star *}$ \\
\hline $\begin{array}{l}\text { ¿La diferencia entre grup } \\
\text { Significación estadística } \\
\text { *־a diferencia entre gru } \\
\text { Significación estadística } \\
\text { IMC = índice de masa } \\
\text { la resistencia a la insulin } \\
\text { de baja densidad, VLDL }\end{array}$ & $\begin{array}{l}\text { se analizó mediante la } \\
<0.05 \text {. } \\
\text { s se analizó mediante la } \\
<0.05 \text {. } \\
\text { oral, HOMA-IR = evalu } \\
\text { HDL = lipoproteína de } \\
=\text { lipoproteína de muy }\end{array}$ & $\begin{array}{l}\text { prueba exacta de Fishe } \\
\text { a prueba U de Mann-Wh } \\
\text { ación del modelo de hon } \\
\text { Itta densidad, LDL-C = li } \\
\text { aja densidad. }\end{array}$ & $\begin{array}{l}\text { itney. } \\
\text { neostasis de } \\
\text { ipoproteína }\end{array}$ \\
\hline
\end{tabular}

paraoxonasa (PON) 1, enzima presente en el núcleo de HDL con efecto protector a la oxidación, y entre SOD y HDL en sujetos con sobrepeso u obesidad y en pacientes con diabetes mellitus ${ }^{18-21}$ y baja actividad de SOD en pacientes con diabetes mellitus con niveles bajos de HDL. ${ }^{22,23}$

Aunado a esto, demostramos que el aumento de $1 \mathrm{nmol} / \mathrm{minuto} / \mathrm{mL}$ en la actividad de GPx3 disminuye el índice en 0.023 unidades, consecuencia de que GPx3 se relaciona negativamente con LDL y positivamente con HDL. Al igual que nosotros, un estudio previo demostró la relación inversa de la actividad de GPx3 con LDL/HDL en pacientes isquémicos masculinos. ${ }^{24}$ Estos resultados podrían explicarse porque GPx3 previene la oxidación de los fosfolípidos, ${ }^{25,26}$ por lo que pudiera prevenir la oxidación de LDL, manteniéndose la afinidad de LDL con su receptor ${ }^{27}$ y porque PON1 
Tabla 2. Asociación del índice LDL/HDL como variable dependiente con marcadores de estrés oxidativo

\begin{tabular}{|c|c|c|c|c|}
\hline & Coeficiente $\boldsymbol{\beta}$ (IC 95\%) & $\begin{array}{l}\text { Coeficiente de } \\
\text { correlación parcial }\end{array}$ & $p$ & $\mathrm{r}^{2}$ ajustada \\
\hline $\mathrm{SOD}(\mathrm{U} / \mathrm{mL})$ & $-1.153(-2.109 a-0.197)$ & -0.098 & 0.018 & 0.008 \\
\hline $\mathrm{SOD}^{*}(\mathrm{U} / \mathrm{mL})$ & $-1.014(-1.930 a-0.099)$ & -0.090 & 0.030 & 0.094 \\
\hline $\mathrm{GP} \times 3(\mathrm{nmol} / \mathrm{minuto} / \mathrm{mL})$ & $-0.024(-0.036 a-0.013)$ & -0.255 & $<0.0005$ & 0.065 \\
\hline $\mathrm{GP} \times 3^{*}(\mathrm{nmol} / \mathrm{minuto} / \mathrm{mL})$ & $-0.023(-0.035 a-0.012)$ & -0.255 & $<0.0005$ & 0.104 \\
\hline $\mathrm{MPO}^{\mathrm{a}}(\mathrm{pg} / \mathrm{dL})$ & $<0.0005(<0.0005$ a 0.0005$)$ & 0.408 & $<0.0005$ & 0.167 \\
\hline $\mathrm{MPO}^{a^{\star}}(\mathrm{pg} / \mathrm{dL})$ & $<0.0005(<0.0005$ a 0.0005$)$ & 0.235 & 0.052 & 0.278 \\
\hline Mg en suero (mg/dL) & $-0.243(-0.634$ a 0.148$)$ & -0.058 & 0.222 & 0.003 \\
\hline $\mathrm{Mg}^{*}$ en suero $(\mathrm{mg} / \mathrm{dL})$ & $-0.339(-0.732$ a 0.054$)$ & -0.081 & 0.090 & 0.055 \\
\hline Mga en uñas $(\mu \mathrm{g} / \mathrm{g})$ & $-0.001(-0.003 a<0.0005)$ & -0.309 & 0.085 & 0.065 \\
\hline Mga* en uñas $(\mu \mathrm{g} / \mathrm{g})$ & $-0.001(-0.003 a<0.0005)$ & -0.437 & 0.020 & 0.351 \\
\hline
\end{tabular}

Tabla 3. Análisis de asociación de oxLDL/HDL como variable dependiente con el índice LDL/HDL y enzimas antioxidantes

\begin{tabular}{|c|c|c|c|c|}
\hline & Coeficiente $\beta$ (IC 95\%) & Coeficiente de correlación parcial & p & $r^{2}$ ajustada \\
\hline LDL/HDL & $0.365(0.271$ a 0.459$)$ & 0.532 & $<0.0005$ & 0.283 \\
\hline LDL/HDL* & $0.831(0.640$ a 1.022$)$ & 0.582 & $<0.0005$ & 0.348 \\
\hline $\mathrm{SOD}(\mathrm{U} / \mathrm{mL})$ & $-2.648(-3.713 a-1.584)$ & -0.383 & $<0.0005$ & 0.146 \\
\hline $\mathrm{SOD}^{*}(\mathrm{U} / \mathrm{mL})$ & $-2.760(-3.837$ a 0.148$)$ & -0.059 & $<0.0005$ & 0.144 \\
\hline GP×3 (nmol/minuto/mL) & $-0.014(-0.022 a-0.006)$ & -0.280 & 0.001 & 0.078 \\
\hline $\mathrm{GP} \times 3^{*}(\mathrm{nmol} / \mathrm{minuto} / \mathrm{mL})$ & $-0.016(-0.024 a-0.008)$ & -0.317 & 0.047 & 0.092 \\
\hline
\end{tabular}

Regresión lineal multivariable. *Ajustada para edad (años), sexo (femenino/masculino), fumador (no/sí), IMC ( $\mathrm{kg} / \mathrm{m}^{2}$ ) y HOMA-IR. Significación estadística p $<0.05 . \mathrm{n}=214$

IC = intervalo de confianza, oxLDL/HDL = índice lipoproteína de baja densidad oxidada/lipoproteína de alta densidad, LDL/HDL = índice lipoproteína de baja densidad/lipoproteína de alta densidad, $\mathrm{SOD}=$ superóxido dismutasa, $\mathrm{GP} \times 3$ = glutation peroxidasa 3 .

y GPx se asocian, ${ }^{19}$ pudiéndose presentar un efecto adicional de modulación entre GPx3 y HDL. Al igual que nuestros resultados, diferentes estudios han reportado la asociación de GPx3 con LDL y HDL. ${ }^{20,22,23,28,29}$

Por otra parte, diversos estudios han propuesto que el índice oxLDL/HDL representa la oxidación lipídica asociada al RCV. ${ }^{30-32}$ En nuestra población, el aumento en una unidad del índice LDL/HDL se tradujo en un aumento de 0.831 del índice oxLDL/HDL, por lo tanto, el índice LDL/HDL representa adecuadamente la presencia de anormalidades oxidativas en las lipoproteínas.

Otro resultado relevante de este estudio fue que el aumento de $1 \mu \mathrm{g}$ de magnesio por gramo de uña se asoció con disminución del índice de 0.001 por unidad. El magnesio es cofactor de lecitina colesterol aciltransferasa y lipoproteína lipasa, por lo que está implicado en la disminución de LDL y el aumento de HDL. De acuerdo con esto, la terapia con magnesio oral aumenta significativa el índice HDL/LDL. ${ }^{33}$ En nuestro estudio, esta asociación no dependió de la relación del magnesio en uñas con las lipoproteínas, sino de otros mecanismos fisiológicos. En este sentido, se conoce el papel fundamental del magnesio en la captación de glucosa en los tejidos sensibles a la insulina ${ }^{34}$ y se ha reportado correlación positiva entre los niveles séricos de magnesio y HDL en pacientes con diabetes tipo $2 .{ }^{35}$ Por otro lado, el magnesio sérico no se asoció con el índice LDL/HDL. Estos resultados controversiales podrían justificarse porque las muestras de uñas son mejores para el análisis de oligoelementos que las muestras de suero, ${ }^{36}$ sin embargo, es necesario realizar estudios para determinar la relación 
entre el magnesio en uñas y el sérico en diferentes poblaciones para esclarecer su asociación.

Finalmente, no observamos asociación entre MPO con el índice LDL/HDL después de controlar el efecto de la edad, sexo, fumar, obesidad y resistencia a la insulina. Se ha reportado que en pacientes con ECV, el aumento del índice MPO/HDL no se acompaña de aumento en los niveles de LDL, ${ }^{37}$ lo cual podría justificar nuestros resultados; además, la asociación de MPO con RCV aparentemente es modulada por el efecto de otros factores metabólicos, por ejemplo, en pacientes diabéticos, la asociación de MPO con ECV es modulada por la concentración de glucosa en sangre. ${ }^{38}$ Aunado a esto, se ha señalado correlación de MPO con edad, talla, peso e IMC en sujetos obesos $\sin \mathrm{ECV}^{39}$

Nuestro estudio tiene algunas limitaciones: es un estudio transversal que no permite establecer una causa-efecto, además no se tomaron en cuenta otras variables confusoras como la dieta y el ejercicio, que podrían modificar el estado de estrés oxidativo de los sujetos de estudio. El tamaño de la muestra es adecuado para un poder estadístico satisfactorio en los análisis con menor número de población, sin embargo, sería conveniente realizar investigaciones adicionales en muestras independientes y más grandes para una futura aplicación en la población.

\section{Conclusiones}

El índice LDL/HDL se asoció inversamente con antioxidantes (SOD, Gpx3) y positivamente con la oxidación de las lipoproteínas (oxLDL/HDL), después de controlar el efecto atribuible a edad, sexo, fumar, obesidad y resistencia a la insulina. Estos hallazgos sugieren conjuntamente que el índice puede considerarse una referencia temprana para el estado de oxidación independiente de otros factores de RCV y estrés oxidativo, además de contribuir a la comprensión de la relación compleja entre el perfil de lípidos y el equilibrio del estado de óxido reducción, independientemente de otros factores de riesgo.

\section{Agradecimientos}

Agradecemos el compromiso y la dedicación de los químicos del laboratorio del Hospital Universitario de Puebla, especialmente a Susana Pérez-Fernández, Julia González Ortega y Gisela Ariza Gallardo.

\section{Bibliografía}

1. Le NA. Lipoprotein-associated oxidative stress: a new twist to the postprandial hypothesis. Int J Mol Sci. 2014:16:401-419.

2. Expert Panel on Detection Evaluation and Treatment of High Blood Cholesterol in Adults. Third Report of the National Cholesterol Education Program (NCEP). Expert panel on Detection Evaluation and Treatment of High Blood Cholesterol in Adults (Adult tratment Panel III) final report. Circulation. 2002;106:3143-3421.

3. Ruscica M, Botta M, Ferri N, Giorgio E, Macchi C, Franceschini G, et al. High density lipoproteins inhibit oxidative stress-induced prostate cancer cell proliferation. Sci Rep. 2018;8:2236.

4. Pawlak K, Mysliwiec M, Pawlak D. Oxidized low-density lipoprotein (oxLDL) plasma levels and oxLDL to LDL ratio. Are they real oxidative stress markers in dialyzed patients? Life Sci. 2013;92:253-258.

5. Linna M, Ahotupa M, Löppönen MK, Irjala K, Vasankari T. Circulating oxidised LDL lipids, when proportioned to HDL-c, emerged as a risk factor of all-cause mortality in a population-based survival study. Age Ageing. 2013;42:110-113.

6. Anatoliotakis N, Deftereos S, Bouras G, Giannopoulos G, Tsounis D, Angelidis $\mathrm{C}$, et al. Myeloperoxidase: expressing inflammation and oxidative stress in cardiovascular disease. Curr Top Med Chem. 2013; 13:115-138.

7. Zheltova AA, Kharitonova MV, lezhitsa IN, Spasov AA. Magnesium deficiency and oxidative stress: an update. Biomedicine (Taipei). 2016; 6:8-14.

8. Rupérez Al, Gil A, Aguilera CM. Genetics of oxidative stress in obesity. Int J Mol Sci. 2014:15:3118-344.

9. Flores-Mateo G, Carrillo-Santisteve P, Elosua R, Guallar E, Marrugat J, Bleys J, et al. Antioxidant enzyme activity and coronary heart disease: meta-analyses of observational studies. Am J Epidemiol. 2009; 170:135-147.

10. Kannel WB. Lipids, diabetes, and coronary heart disease: insights from the Framingham Study. Am Heart J. 1985;110:1100-1107.

11. Millán J, Pintó X, Muñoz A, Zúñiga M, Rubiés-Prat J, Pallardo LF, et al. Lipoprotein ratios: physiological significance and clinical usefulness in cardiovascular prevention. Vasc Health Risk Manag. 2009;5:757-765.

12. Kunutsor SK, Zaccardi F, Karppi J, Kurl S, Laukkanen JA. Is high serum $\mathrm{LDL} / \mathrm{HDL}$ cholesterol ratio an emerging risk factor for sudden cardiac death? Findings from the KIHD Study. J Atheroscler Thromb. 2017; 24:600-608.

13. Ormazabal V, Nair S, Elfeky O, Aguayo C, Salomon C, Zúñiga FA. Association between insulin resistance and the development of cardiovascular disease. Cardiovasc Diabetol. 2018;17(1):122.

14. Londoño-Pérez C, Rodríguez-Rodríguez I, Gantiva-Diaz CA, et al. Cuestionario para la clasificación de consumidores de cigarrillo (C4) para jóvenes. Perspect Psicol. 2011;7:281-291.

15. Babor TF, Higgins-Biddle JC, Saunders JB, Monteiro MG. AUDIT. The alcohol use disorders identification test. Guidelines for use in primary care. Suiza: World Health Organization/Department of Mental Health and Substance Dependence; 2001.

16. Levy JC, Matthews D, Hermans MP. Correct homeostasis model assessment (HOMA) evaluation uses the computer program. Diabetes Care. 1998;21:2191-2192.

17. López-González ÁA, Rivero-Ledo YI, Vicente-Herrero MT, Gil-Llinás M, Tomás-Salvá M, Riutord-Fe B. Índices aterogénicos en trabajadores de diferentes sectores laborales del área mediterránea española. Clin Investig Arterioscler. 2015;27:118-128.

18. Kumar S, Batra J, Arora M. Correlation of HDL associated paraoxonase 1 with oxidative stress markers in hypertensive dyslipidemic patients. J Clin Diagnostic Res. 2018;12:BC23-BC26.

19. Sarhat ER, Sarhat TR. Study of serum levels of melatonin, paraoxonase, oxidative stress in Iraqi patients with acute myocardial infarction. Eur Acad Res. 2016;1:112-131.

20. Báez-Duarte BG, Zamora-Ginez I, De Jésus KL, Torres-Rasgado E, González-Mejía ME, Porchia L, et al. Association of the metabolic syndrome with antioxidant defense and outstanding superoxide dismutase activity in Mexican subjects. Metab Syndr Relat Disord. 2016;14:154-160.

21. Rajprabha, Hamid M, KumarMeena R, Syedyawer H. Study of antioxidant enzyme-superoxide dismutase activity and lipid profile in diabetes mellitus patients. Int J Healthc Biomed Res. 2014;2:22-29.

22. Hisalkar PJ, Patne $A B$, Fawade MM. Assessment of plasma antioxidant levels in type 2 diabetes patients. Int Biol Med Res. 2012;3:1796-1800.

23. Humaira M, Raqeeb A, Memon A, Khoharo HK. Malondialdehyde, blood lipids and antioxidant activity in newly diagnosed type 2 diabetics. J Liaquat Uni Med Heal Sci. 2016;15:78-82.

24. Pioruńska-Stolzmann M, Batko J, Majewski W. Lipid profile, lipase and glutathione peroxidase activities in the serum of patients with atherosclerosis. Med Sci Monit. 1999:5:900-903.

25. Arthur JR. The glutathione peroxidases. Cell Mol Life Sci. 2001;57:1825-1835.

26. Yoshida H, Kisugi R. Mechanisms of LDL oxidation. Clin Chim Acta. 2010;411:1875-1882. 
27. Levitan I, Volkov S, Subbaiah PV. Oxidized LDL: diversity, patterns of recognition, and pathophysiology. Antioxid Redox Signal. 2010;13:39-75.

28. Buijsse B, Lee DH, Steffen L, Erickson RR, Luepker RV, Jacobs DR et al. Low serum glutathione peroxidase activity is associated with increased cardiovascular mortality in individuals with low HDLc's. PLoS One. 2012;7:e38901.

29. Gawlik K, Naskalski JW, Fedak D, Pawlica-Gosiewska D, Grudzień U, Dumnicka $P$, et al. Markers of antioxidant defense in patients with type 2 diabetes. Oxid Med Cell Longev. 2016;2016:2352361.

30. Harmon ME, Campen MJ, Miller C, Shuey C, Cajero M, Lucas S, et al. Associations of circulating oxidized LDL and conventional biomarkers of cardiovascular disease in a cross-sectional study of the Navajo population. PLoS One. 2016;11:e0143102.

31. Huang H, Mai W, Liu D, Hao Y, Tao J, Dong Y. The oxidation ratio of LDL: a predictor for coronary artery disease. Dis Markers. 2008; 24:341-349.

32. Wang A, Li S, Zhang N, Dai L, Zuo Y, Wang Y, et al. Oxidized low-density lipoprotein to high-density lipoprotein ratio predicts recurrent stroke in minor stroke or transient ischemic attack. Stroke. 2018;49:2637-2642.
33. Shechter M. Magnesium and cardiovascular system. Magnes Res. 2010;23:1-13.

34. Kim DJ, Xun P, Liu K, Loria C, Yokota K, Jacobs DR, et al. Magnesium Intake in Relation to Systemic Inflammation, Insulin Resistance, and the Incidence of Diabetes. Diabetes Care. 2010;33:2604-2610.

35. Chutia H, Lynrah KG. Association of serum magnesium deficiency with insulin resistance in type 2 diabetes mellitus. J Lab Physicians. 2015;7:75-78.

36. Nasli-Esfahani E, Faridbod F, Larijani B, Ganjali MR, Norouzi P. Trace element analysis of hair, nail, serum and urine of diabetes mellitus patients by inductively coupled plasma atomic emission spectroscopy. J Diabetes Metabol Disord. 2011;10:1-9.

37. Kimak E, Zięba B, Duma D, Solski J. Myeloperoxidase level and inflammatory markers and lipid and lipoprotein parameters in stable coronary artery disease. Lipids Health Dis. 2018;17:71.

38. Song $\mathrm{P}, \mathrm{Xu}$ J, Song $\mathrm{Y}$, Jiang $\mathrm{S}$, Yuan $\mathrm{H}$, Zhang X. Association of plasma myeloperoxidase level with risk of coronary artery disease in patients with type 2 diabetes. Dis Markers. 2015;2015:1-5.

39. Zubair-Nagi F, Naseem T, Yasmin R. A study of myeloperoxidase and lipid profile in obese individuals. Polish J Manag Stud. 2014;8:726-729. 